\title{
The impact of exercise intensity on the release of cardiac biomarkers in marathon runners
}

\author{
Alejandro Legaz-Arrese - Keith George • Luis Enrique Carranza-García • \\ Diego Munguía-Izquierdo • Teresa Moros-García $\cdot$ Enrique Serrano-Ostáriz
}

Received: 22 August 2010/Accepted: 23 February 2011

(C) Springer-Verlag 2011

\begin{abstract}
We sought to determine the influence of exercise intensity on the release of cardiac troponin I (cTnI) and $\mathrm{N}$-terminal pro-brain natriuretic peptide (NT-proBNP) in amateur marathon runners. Fourteen runners completed three exercise trials of the same duration but at exercise intensities corresponding to: (a) a competitive marathon [mean $\pm \mathrm{SD}$ : heart rate $159 \pm 7$ beat $\mathrm{min}^{-1}$, finish time $202 \pm 14 \mathrm{~min}]$; (b) $95 \%$ of individual anaerobic threshold [heart rate $144 \pm 6$ beat $\mathrm{min}^{-1}$ ] and; (c) $85 \%$ of individual anaerobic threshold [heart rate $129 \pm 5$ beat $\mathrm{min}^{-1}$ ]. cTnI and NT-proBNP were assayed from blood samples collected before, $30 \mathrm{~min}$ and $3 \mathrm{~h}$ post-exercise for each trial. cTnI and NT-proBNP were not different at baseline before each trial. After exercise at $85 \%$ of individual anaerobic threshold cTnI was not significantly elevated. Conversely, cTnI was elevated after exercise at $95 \%$ of individual anaerobic threshold $\left(0.016 \mu \mathrm{g} \mathrm{L}^{-1}\right)$ and to an even greater
\end{abstract}

Communicated by Dag Linnarsson.

A. Legaz-Arrese · T. Moros-García · E. Serrano-Ostáriz Section of Physical Educations and Sports,

University of Zaragoza, Saragossa, Spain

K. George

Research Institute for Sport and Exercise Sciences,

Liverpool John Moores University, Liverpool, UK

L. E. Carranza-García ( $₫)$

Facultad de Organización Deportiva,

Universidad Autónoma de Nuevo León,

Av. Universidad s/n, Cd. Universitaria,

66455 San Nicolás de los Garza, Nuevo León, Mexico

e-mail: kique_79@hotmail.com

D. Munguía-Izquierdo

Section of Physical Education and Sports,

University Pablo de Olavide, Seville, Spain extent after exercise at competition intensity $\left(0.054 \mu \mathrm{g} \mathrm{L}^{-1}\right)$. Peak post-exercise values of NT-proBNP were elevated to a similar extent after all exercise trials $(P<0.05)$. The upper reference limit for $\mathrm{cTnI}$ $\left(0.04 \mu \mathrm{g} \mathrm{L}^{-1}\right)$ was exceeded in six subjects at competition intensity. No data for NT-proBNP surpassed its upper reference limit. Peak post-exercise values for cTnI and NTproBNP were correlated with their respective baseline values. These data suggest exercise intensity influences the release of cTnI, but not NT-proBNP, and that competitive marathon running intensity is required for $\mathrm{cTnI}$ to be elevated over its upper reference limit.

Keywords cTnI - NT-proBNP · Cardiac damage . Amateur runners

\section{Introduction}

The beneficial effects of moderate intensity physical activity for the cardiovascular system are well documented (Ignarro et al. 2007; Wendel-Vos et al. 2004). Whilst many people follow this type of exercise prescription, a sizeable number of amateur "athletes" engage in strenuous training and competitive activity whose exercise intensity and duration clearly exceed the standards programs established by various organizations for healthy physical activity (Haskell et al. 2007). Whether the elevated exercise volume completed by amateur "athletes" provides additional health benefits or, controversially, increased health risk is currently not clear. Indeed, the impact of years of highintensity training and competition upon cardiovascular health has been the focus of recent studies and comment (Bhella and Levine 2010; Pelliccia et al. 2010). The debate about the cardiovascular health consequences of strenuous 
exercise completed over many years of training and competition has largely arisen because of the common observation of elevated cardiac biomarkers after competitive prolonged endurance exercise (Regwan et al. 2010; Scharhag et al. 2008). Post-exercise values for serum troponins (cTnI and cTnT) and N-terminal pro BNP (NT-proBNP), which are accepted parameters for the identification of cardiac damage and dysfunction, are often higher than the population upper reference limit (URL) in a high percentage of athletes in these strenuous competitions (Neilan et al. 2006; Serrano-Ostáriz et al. 2009).

The short and long-term clinical significance of an increase in cardiac-specific biomarkers following strenuous endurance exercise in healthy athletes is unclear. Some have suggested that there might be an optimum exercise intensity/duration with respect to the impact of physical activity on cardiovascular health (La Gerche and Prior 2007; Whyte 2008). Despite this speculation much remains unknown with respect to the acute effect of exercise of varying intensity has on the appearance of cardiac biomarkers. In a well-controlled, repeated measures design (Serrano-Ostáriz et al. 2011), the appearance of cTnI postexercise was associated with exercise intensity but this did not include competitive performance intensities, which is common in field studies, and thus may have underestimated the impact of exercise intensity on biomarker release. The aim, therefore, of the current study was to extend our understanding of the impact of the intensity of prolonged running exercise on the exercise-related release in cTnI and NT-proBNP in a single cohort of amateur endurance runners.

\section{Materials and methods}

Subjects and design

Fourteen male runners (age, $42 \pm 8$ years; height, $1.76 \pm$ $0.06 \mathrm{~m}$; body mass, $74.3 \pm 5.7 \mathrm{~kg} ; V_{2} \max , 56.4 \pm 5.0 \mathrm{ml}$ $\mathrm{kg}^{-1} \min ^{-1}$; training volume, $67 \pm 14 \mathrm{~km}^{\text {week }}{ }^{-1}$ ) scheduled to participate in the 2009 Zaragoza marathon race were recruited by an open invitation linked to the official race website. After an initial meeting where the aims and protocol of the research were explained all subjects provided written informed consent. This study was approved by the Research Ethics Committee of the Aragón (Spain) Government.

To exclude acute or chronic diseases, an initial medical examination including a self-report history of cardiac symptoms was followed by the assessment of resting blood pressure, resting 12-lead electrocardiography (ECG) and a standard echocardiogram. In addition, $V \mathrm{O}_{2 \max }$ and the individual anaerobic threshold (IAT) were determined during a graded treadmill exercise test to volitional exhaustion. To determine the IAT using the method reported by Stegmann et al. (1981), incremental multistage treadmill ergometry (Laufergotest, Jaeger, Germany) was started at $8 \mathrm{~km} \mathrm{~h}^{-1}$ and increased by $2 \mathrm{~km} \mathrm{~h}^{-1}$ every 3 min until exhaustion. Capillary blood samples were taken from the hyperaemized earlobe at the end of each stage and $1,3,5,7,10$ and $20 \mathrm{~min}$ after cessation of exercise to determine lactate concentrations (1500 sport YSI, Yellow Springs, Ohio, USA). In addition, a 12-lead ECG, blood pressure and $V \mathrm{O}_{2 \max }(\mathrm{CPX} / \mathrm{MAX}$ MedGraphics, St. Paul, Minnesota, USA) were recorded.

Initially, all subjects completed the 2009 Zaragoza Marathon. Average heart rate (HR) was recorded, as was the time taken to complete the marathon. All the athletes completed the marathon as quickly as possible. Over the following 2 weeks the athletes took part in two exercise sessions (in randomized order) at intensities of 85 and 95\% of IAT on a natural circuit (distance, 4,600 m; maximum slope, $2 \%$ ). The duration of both sessions was equal to the time required for each athlete to complete the marathon race. Exercise at 95\% IAT has been used previously to represent the exercise intensity that athletes could maintain without excessive fatigue for a period of $3 \mathrm{~h}$ (Serrano-Ostáriz et al. 2011). Exercise at 85\% IAT was set to represent an easier "training" run where HR would be significantly lower than both competition pace and $95 \%$ IAT. Intensities were determined by heart rates and checked by heart rate monitoring of the submaximal bouts (Polar, Kempele, Finland). For the sessions performed at 85 and $95 \%$ IAT, a maximum variation of \pm 2 beat $\min ^{-1}$ was allowed between the prescribed heart rate and the real measured values. We choose to maintain 85 and $95 \%$ of IAT for the same duration as the Marathon race so as not to implicate exercise duration as a confounding factor. We appreciate, a priori, that this would result in exercise trials of different volume but understand that not all facets of exercise can be controlled simultaneously.

Runners were advised to avoid strenuous exercise 2 days before each trial. Venous blood samples to measure cardiac biomarkers were taken before, $30 \mathrm{~min}$ and $3 \mathrm{~h}$ after each exercise trial. All blood draws were taken from an antecubital vein. Samples were quickly centrifuged and the serum was drawn off and stored below $-80^{\circ} \mathrm{C}$. NT-proBNP was measured by Elecsys proBNP chemiluminescence immunoassay technology on an automated analyser (Elecsys proBNP; Elecsys 2010; Roche Diagnostics, Lewes, Sussex). The URL for NT-proBNP was $125 \mathrm{ng} \mathrm{L}^{-1}$ (Al-Barjas et al. 2004). cTnI was measured by Access AccuTnI assay (Beckman Coulter, Fullerton, California). The URL for cTnI, defined as the 99th percentile of healthy participants, was $0.04 \mu \mathrm{g} \mathrm{L}^{-1}$ (Apple et al. 2003). 
Statistical analysis

Statistical analyses were performed using the Statistical Package of Social Sciences (SPSS), version 15.0. Data are expressed as mean $\pm \mathrm{SD}$. A comparison of baseline data for $\mathrm{cTnI}$ and NT-proBNP was made via repeated measures one-way ANOVA. The impact of exercise intensity and sample time were assessed using a repeated measures 2-way ANOVA. The delta score (baseline to peak postexercise values) was compared between exercise intensities by repeated measures one-way ANOVA. Pearson product moment correlations were used to associate absolute peak and delta [baseline - peak] scores for cTnI and NTproBNP with baseline data, age, exercise HR, training volume, $V \mathrm{O}_{2 \max }$ and velocity at IAT. To control the possible trial "order effect", a repeated measures one-way ANOVA was completed to compare the peak post-exercise values of cTnI and NT-proBNP from the two exercise sessions (in randomized order) at intensities of 85 and $95 \%$ of IAT. Critical alpha was set at $P=0.05$.

\section{Results}

Marathon performance and HR data and exercise test performance (IAT)

The athletes ran the marathon in $202 \pm 14$ min (range 184-231 min) with an average HR of $159 \pm 7$ beat min $^{-1}$ $(90 \pm 4 \%$ HRmax $)$. The IAT was identified at $11.9 \pm$ $1.2 \mathrm{~km} \mathrm{~h}^{-1}$; HR, $151 \pm 7$ beat $\min ^{-1}$; \%HRmax, $85 \pm 4$; $\% \mathrm{VO}_{2} \max , 70 \pm 11$. For subsequent trials, HR at 95 and $85 \%$ IAT of the IAT was established as $144 \pm 6$ beat $\min ^{-1}(81 \pm 3 \% \quad$ HRmax $)$ and $129 \pm 5$ beat $\min ^{-1}$ (73 $\pm 3 \%$ HRmax), respectively.

\section{Prolonged exercise trials}

All runners completed all exercise trials at the prescribed intensities. As expected, HR was significantly altered depending upon exercise intensity $(P<0.001)$, and the distance covered at $85 \%$ IAT $(33.2 \pm 1.9 \mathrm{~km})$ and $95 \%$ IAT $(38.1 \pm 2.3 \mathrm{~km})$ was significantly less than the marathon distance $(P<0.001)$.

Pre-exercise values of cTnI were not significantly different for all trials $(P=0.581)$. From the 2-way ANOVA a significant main effect of assessment time (pre, 0.011; post $30 \mathrm{~min}, 0.014$; post $\left.3 \mathrm{~h}, 0.027 \mu \mathrm{g} \mathrm{L}^{-1} ; P=0.002\right)$ confirmed an exercise response (Table 1). Peak post-exercise cTnI values after the $85 \%$ IAT trial rose only marginally $(P>0.05)$. Peak cTnI levels increased significantly after exercise at an intensity of 95\% IAT $(P=0.023)$ and at competition intensity $(P=0.001)$. Analysis of $\mathrm{cTnI}$ data post-exercise identified a significant main effects for intensity $(P=0.037)$ of trial but no significant interaction of test time and exercise intensity $(P=0.069)$. Peak postexercise values of cTnI were higher than the URL in six runners $(43 \%)$, but only after the exercise at competition intensity. A significant relationship between baseline and post-exercise levels was observed at 85 and $95 \%$ IAT ( $R=0.60$ and 0.72 , respectively; $P<0.05$ ), but not at competition intensity $(R=0.50, P=0.068)$. Peak postexercise values of cTnI after the exercise at competition intensity were positively related with average HR $(R=0.65, P=0.013)$ and negatively related with age $(R=-0.65, P=0.012)$. The association between age and peak post-exercise values of cTnI was not significant when average HR and baseline values of cTnI were controlled $(R=-0.36, P=0.252)$.

Pre-exercise values of NT-proBNP were not significantly different for all trials $(P=0.607)$. From the 2 -way ANOVA a significant main effect of assessment time (pre, 13.19; post $30 \mathrm{~min}, 38.81$; post $3 \mathrm{~h}, 35.48 \mathrm{ng} \mathrm{L}^{-1}$; $P<0.001$ ) confirmed an exercise response (Table 1), but no sample surpassed the URL. A non-significant main effect of exercise intensity as well as interaction term was observed for NT-proBNP $(P=0.218)$. A significant relationship between baseline NT-proBNP levels and postexercise values $(R=0.72$ and 0.89 , respectively; $P<0.05)$ during the three sessions was also established. Peak post-exercise values of NT-proBNP after the exercise at competition intensity were related with average $\mathrm{HR}$ ( $R=0.66, P=0.010)$. For the two exercise trials performed at randomization to 85 and $95 \%$ of IAT, there were no significant differences in the peak post-exercise values of NT-proBNP (first trial, 37.42; second trial, $34.20 \mathrm{ng} \mathrm{L}^{-1} ; P=0.541$ ) and cTnI (first trial, 0.016; second trial, $\left.0.015 \mu \mathrm{g} \mathrm{L}^{-1} ; P=0.896\right)$.

\section{Discussion}

This study demonstrates that exercise intensity, from training run speed to competition speed, has a differential effect on post-exercise appearance of the cardiac biomarkers cTnI and NT-proBNP. Specifically, prolonged exercise at competition intensity results in a substantial increase in $\mathrm{cTnI}$ post-exercise, compared to lower intensity running speeds, and is the only exercise intensity that produces post-exercise values of cTnI over the URL. By comparison, the impact of exercise intensity upon NTproBNP is negligible.

Specifically, we observed that running at a moderate (less than IAT) resulted in small changes in biomarkers with no athletes producing values that exceeded the URL of cTnI or NT-proBNP. This supports previous findings for 
Table 1 Cardiac biomarkers levels before and after exercise sessions

\begin{tabular}{|c|c|c|c|c|c|c|}
\hline & \multicolumn{3}{|l|}{$\operatorname{cTnI}\left(\mu \mathrm{g} \mathrm{L}^{-1}\right)$} & \multicolumn{3}{|c|}{ NT-proBNP (ng L $\left.{ }^{-1}\right)$} \\
\hline & Pre-exercise & 30 min post & $3 \mathrm{~h}$ post & Pre-exercise & 30 min post & $3 \mathrm{~h}$ post \\
\hline $85 \%$ IAT & $0.009 \pm 0.01$ & $0.013 \pm 0.01$ & $0.012 \pm 0.01$ & $11 \pm 8$ & $32 \pm 22 *$ & $34 \pm 23^{*}$ \\
\hline $95 \%$ IAT & $0.011 \pm 0.01$ & $0.011 \pm 0.01$ & $0.016 \pm 0.01$ & $13 \pm 7$ & $32 \pm 14^{*}$ & $34 \pm 16^{*}$ \\
\hline Competition & $0.013 \pm 0.01$ & $0.017 \pm 0.01$ & $0.054 \pm 0.06^{*, \#}$ & $15 \pm 11$ & $52 \pm 31 *$ & $38 \pm 24^{*}$ \\
\hline
\end{tabular}

For cTnI, significant main effect for test time and exercise intensity but not significant interaction. For NT-proBNP, significant main effect for test time but not for exercise intensity

IAT individual anaerobic threshold

* Significant different from pre-exercise values, ${ }^{*}$ significant different from other exercise intensity

shorter exercise durations (Fu et al. 2009; Serrano-Ostáriz et al. 2011) and the indirect evidence from a recent metaanalysis (Shave et al. 2007b). When running the marathon at competition intensity (HR difference of c. 15 beats $\min ^{-1}$ ) 6 out of 14 athletes exceeded the URL for cTnI. Single competitive event studies would support a similar frequency of cTnI values above the URL (Shave et al. 2007a). The increase in cTnI across the running speeds/ intensities employed in the current repeated measures study was not necessarily linear and suggests that there may be a threshold exercise intensity where cTnI release becomes more marked. This is important to note for those trying to interpret post-exercise cTnI elevations in any clinical setting (Whyte 2008). Whether the release of cTnI has an exponential relationship with exercise across a broader and higher range of exercise intensities is yet to be conclusively determined. Indeed, not all cTnI data in the current study exceeded the URL after the completion of the competitive marathon. Numerous studies have reported a sporadic release of cTn after marathon exercise as well as differences in the magnitude of post-exercise cTn serum concentrations (Regwan et al. 2010). Shave et al. (2010) recently described cTnI release after $30 \mathrm{~min}$ of short duration high-intensity exercise in 6 out of 8 runners and noted that the responses were markedly heterogenous. Consequently, available data implies that other personal and research design-related factors, in addition to intensity, may influence the data obtained for cardiac biomarkers post-exercise in athletes (as well as whether this release would exceed the URL). These points require further study.

The mechanism(s) of the exercise-induced release of cardiac troponins and the clinical and/or performance implications are still being debated. Although we did not perform cardiovascular imaging studies alongside blood draws in the current study, recent empirical studies (Wilson et al. 2010) and broader reviews (Oxborough et al. 2010) do not provide compelling evidence that biomarker release and changes in left ventricular function post-exercise are causally related. Whether serum cTn observed post-exercise reflects reversible or irreversible myocardial damage is also still to be conclusively determined. However, the time course and magnitude of post-exercise cTn kinetics do not closely resemble the kinetics of cTn release in ACS (Middleton et al. 2008). Most athletes with elevated cTn post-exercise present with no other signs and symptoms of coronary disease and have mostly completed multiple years of training and numbers of competitive races without a significant cardiac event (Scharhag et al. 2008). Moreover, recent data suggest that strenuous endurance exercise improves life expectancy (Ruiz et al. 2011). Therefore, it is tempting to suggest that the appearance of cTnI after physical exercise does not reflect a clinically threatening (irreversible) myocardial injury, but rather may reflect a reversible increase in cellular membrane permeability and rapid troponin release from the cytosolic pool post-exercise (Lippi and Plebani 2009). Some authors have suggested that exercise-induced cTn release reflects the activation of cellular cascades that result in cardiac hypertrophy (Scharhag et al. 2006). Changes in cellular membrane permeability, subsequent to an increased rate and force of cardiac contraction during endurance exercise, may provide a mechanism by which cytosolic troponin is released into the circulation after exercise. An explanation as to why exercise intensity mediates this process can, from the current data, only be speculative at this stage. However, highintensity exercise results in an increased production of oxygen free radicals that may lead to membrane disruption and, hence, cTn release. This theory has gained some support in a recent study in an animal model that demonstrated a temporal association of elevations in serum cardiac troponin $\mathrm{T}$ and myocardial oxidative stress after prolonged exercise (Nie et al. 2010). An increase in myocardial sarcolemmal permeability might be due to other exercise-intensity-dependent factors, such as altered acidbase balance (Shave et al. 2010), dehydration, and hemoconcentration (George et al. 2004). Recently, it was also suggested that cTn may be released in response to transient ischemia alone, without necrosis. It was suggested that during ischemia, cardiac myocytes develop blebs on the surface of their plasma membrane and release cytoplasmic 
contents (Hickman et al. 2010). Increasing exercise intensity in this scenario would likely increase the relative amount of hypoxia and thus bleb formation. At the moment these theories have no substantive evidence to support them but are worthy of further investigation.

The current data also confirms a relationship between peak post-exercise cTnI and baseline levels (SerranoOstáriz et al. 2011). A key factor that may influence peak post-exercise biomarker concentration, and thus the percentage of individuals exceeding the cTnI URL, is number of samples taken during the post-exercise recovery period (Middleton et al. 2008; Shave et al. 2010). Middleton et al. (2008) showed that after a marathon on a treadmill all eight subjects showed a post-exercise release of cTnI that peaked at different time points post-exercise. Other factors like age, sex, and training status may influence peak postexercise cTnI values at competition intensity. To our knowledge no well-designed study has monitored the effects of these factors on peak post-exercise biomarker concentration.

Previous studies have suggested that an increase in NTproBNP is fundamentally associated with exercise duration (Serrano-Ostáriz et al. 2011). Given that we "clamped" exercise duration across a range of exercise intensities (and thus employed different volumes of exercise) yet still observed similar post-exercise NT-proBNP release, our data support this proposal. Given that NT-proBNP is released in response to cardiac work and "myocardial tissue stretch" it is pertinent to consider why NT-proBNP release post-exercise is not intensity dependent. Whilst speculative, it may reflect the fact that preload changes in the heart do not increase with exercise intensity in a linear fashion. Due to the fact that increasing exercise intensity presents a ventricular filling (and preload stretch) limitation as $\mathrm{R}-\mathrm{R}$ interval decreases it is possible that the rate of NTproBNP release is maximized at HR of $100-120$ beats $\min ^{-1}$ as preload reaches a maximum and after this point exercise duration exerts a bigger influence on NT-proBNP release.

The increase in NT-proBNP with exercise has also been associated with training status (Herrmann et al. 2003; Neilan et al. 2006) and exercise duration (Serrano-Ostáriz et al. 2011). Both these factors could partially explain why, in contrast to other studies of marathon runners (Herrmann et al. 2003; Neilan et al. 2006; Scharhag et al. 2005; Trivax et al. 2010; Wilson et al. 2010), none of our athletes exceeded the URL for NT-proBNP. Our athletes completed the marathon in less time (average $202 \mathrm{~min}$ ) than those in the above-mentioned studies (range average 209-256 min). This study demonstrated, as have others (Sahlén et al. 2008; Serrano-Ostáriz et al. 2011), that athletes' postexercise NT-proBNP is strongly associated with their respective baseline levels. Baseline values for NT-proBNP for our athletes (average $15 \mathrm{ng} \mathrm{L}^{-1}$ ) were clearly less than those found in some previous studies (Herrmann et al. 2003; Neilan et al. 2006; Scharhag et al. 2005; Trivax et al. 2010; Wilson et al. 2010) in marathon runners (range average 37-63 $\mathrm{ng} \mathrm{L}^{-1}$ ) which could also explain the lower exercise response in the current study. Given that the resting NT-proBNP data demonstrated high reproducibility even after a 3-year period (Sahlén et al. 2008), these results suggest a more complex interaction of baseline, prior exercise and exercise-related factors that mediate cardiac biomarker responses to prolonged exercise. In the general population, the baseline NT-proBNP value is influenced by age, sex, obesity, malnutrition, volume overload, pulse rate, blood pressure, and prior heart failure (Chen et al. 2008). These factors may explain some of the difference in baseline NT-proBNP between our athletes and those found in previous studies. Between study differences may also reflect variability in detection levels for specific assays. Further exploratory research in a broad range of normal and athletic groups is suggested.

The current study design manipulated running speed, a surrogate of exercise intensity [including competition level performance] and assessed cardiac biomarker release during recovery. Two limitations to the design employed are worthy of comment. Firstly, we "clamped" exercise duration because of its postulated effects on cardiac biomarker release post-exercise (Scharhag et al. 2008). A consequence of this is a progression in total exercise volume with increasing exercise intensity. Clamping both exercise volume and duration whilst manipulating intensity is impossible and thus we made the decision, a priori, based on desire to clamp duration but would support further studies where intensity is manipulated and exercise volume is "clamped", possibly by looking at energy expenditure or heart rate. Inherent to the study design, the exercise duration from the initial competitive marathon race was used for the follow-up low-intensity trials so that the "order effect" could influence the results. However, we observed similar cardiac biomarker values for the two exercise trials performed at random to 85 or $95 \%$ of IAT; therefore, the "order effect" can be considered negligible. Secondly, no blood samples were taken during exercise (Middleton et al. 2008) and thus a peak exercise response and its association with exercise intensity could not be assessed. Again, ongoing studies should address this issue.

In conclusion, our results show that when exercise duration is clamped, there is a significant impact of exercise intensity on the peak post-exercise values of cTnI. This association was not observed for NT-proBNP. We observed that exercise intensity, and potentially baseline cTnI, are the key factors when assessing the likelihood of data exceeding the URL and this is of value to those assessing cTnI in a clinical environment when the subject 
has a performed a recent bout of competitive exercise (i.e., a marathon run).

Acknowledgments We greatly appreciate Dr. Vicente Pej Rodriguez (Grande Covián Medical Center, Zaragoza, Spain), and Drs. Francisco López Alcutan, Miguel Angel Anson Manso (Lozano Blesa Universitary Clinic Hospital, Zaragoza, Spain) for their excellent technical collaboration. Financial support for this study was provided by the Department of Science and Technology, Government of Aragon and Ministry of Science and Innovation of the Spanish Government (DEP 2010-16767).

\section{References}

Al-Barjas M, Nair D, Ayrton P, Morris R, Davar J (2004) How can the role of $\mathrm{N}$ terminal pro $\mathrm{B}$ natriuretic peptide (NT-proBNP) be optimised in heart failure screening? A prospective obsevational comparative study. Eur Heart J Fail 3(Suppl 1):51. doi:10.1016/ S1567-4215(04)90150-6

Apple FS, Quist HE, Doyle PJ, Otto AP, Murakami MM (2003) Plasma 99th percentile reference limits for cardiac troponin and creatine kinase $\mathrm{MB}$ mass for use with European Society of Cardiology/American College of Cardiology consensus recommendations. Clin Chem 49:1331-1336. doi:10.1373/49.8.1331

Bhella PS, Levine BD (2010) The heart of a champion. J Am Coll Cardiol 55:1626-1628. doi:10.1016/j.jacc.2009.12.030

Chen Y, Yao CL, Shimada T (2008) Factors associated plasma B type natriuretic peptide and $\mathrm{N}$-terminal pro-B-type natriuretic peptide levels. Zhonghua Xin Xue Guan Bing Za Zhi 36:825-830

Fu F, Nie J, Tong TK (2009) Serum cardiac troponin T in adolescent runners: effects of exercise intensity and duration. Int J Sports Med 30:168-172. doi:10.1055/s-0028-1104586

George K, Whyte G, Stephenson C, Shave R, Dawson E, Edwards B, Gaze D, Collinson P, George K (2004) Postexercise left ventricular function and $\mathrm{cTnT}$ in recreational marathon runners. Med Sci Sports Exerc 36:1709-1715. doi:10.1249/01.MSS.000 0142408.05337.49

Haskell WL, Lee IM, Pate RR, Powell KE, Blair SN, Franklin BA, Macera CA, Heath GW, Thompson PD, Bauman A (2007) Physical activity and public health: updated recommendation for adults from the American College of Sports Medicine and the American Heart Association. Med Sci Sports Exerc 39:14231434. doi:10.1249/mss.0b013e3180616b27

Herrmann M, Scharhag J, Miclea M, Urhausen A, Herrmann W, Kindermann W (2003) Post-race kinetics of cardiac troponin T and I and N-terminal pro-brain natriuretic peptide in marathon runners. Clin Chem 49:831-834. doi:10.1373/49.5.831

Hickman PE, Potter JM, Aroney C, Koerbin G, Southcott E, Wu AH, Roberts MS (2010) Cardiac troponin may be released by ischemia alone, without necrosis. Clin Chim Acta 411:318323. doi:10.1016/j.cca.2009.12.009

Ignarro LJ, Balestrieri ML, Napoli C (2007) Nutrition, physical activity, and cardiovascular disease: an update. Cardiovasc Res 73:326-340. doi:10.1016/j.cardiores.2006.06.030

La Gerche A, Prior DL (2007) Exercise-is it possible to have too much of a good thing? Heart Lung Circ 16(Suppl 3):S102-S104. doi:10.1016/j.hlc.2007.03.014

Lippi G, Plebani M (2009) High-sensitive troponin testing and the "runner's syndrome". J Emerg Med Aug 12. [Epub ahead of print]. doi:10.1016/j.jemermed.2009.07.005

Middleton N, George K, Whyte G, Gaze D, Collinson P, Shave R (2008) Cardiac troponin $\mathrm{T}$ release is stimulated by endurance exercise in healthy humans. J Am Coll Cardiol 52:1813-1814. doi:10.1016/j.jacc.2008.03.069
Neilan TG, Januzzi JL, Lee-Lewandrowski E, Ton-Nu TT, Yoerger DM, Jassal DS, Lewandrowski KB, Siegel AJ, Marshall JE, Douglas PS, Lawlor D, Picard MH, Wood MJ (2006) Myocardial injury and ventricular dysfunction related to training levels among nonelite participants in the Boston marathon. Circulation 114:2325-2333. doi:10.1161/CIRCULATIONAHA.106.647461

Nie J, Close G, George KP, Tong TK, Shi Q (2010) Temporal association of elevations in serum cardiac troponin $\mathrm{T}$ and myocardial oxidative stress after prolonged exercise in rats. Eur $\mathrm{J}$ Appl Physiol 110:1299-1303. doi:10.1007/s00421-010-1604-6

Oxborough D, Birch K, Shave R, George K (2010) "Exercise-induced cardiac fatigue" - a review of the echocardiographic literature. Echocardiography 27:1130-1140. doi:10.1111/j.1540-8175.2010. 01251.x

Pelliccia A, Kinoshita N, Pisicchio C, Quattrini F, Dipaolo FM, Ciardo R, Di Giacinto B, Guerra E, De Blasiis E, Casasco M, Culasso F, Maron BJ (2010) Long-term clinical consequences of intense, uninterrupted endurance training in Olympic athletes. J Am Coll Cardiol 55:1619-1625. doi:10.1016/j.jacc.2009. 10.068

Regwan S, Hulten EA, Martinho S, Slim J, Villines TC, Mitchell J, Slim AM (2010) Marathon running as a cause of troponin elevation: a systematic review and meta-analysis. J Interv Cardiol 23:443-450. doi:10.1111/j.1540-8183.2010.00575.x

Ruiz JR, Morán M, Arenas J, Lucia A (2011) Strenuous endurance exercise improves life expectancy: it's in our genes. Br J Sports Med 45:159-161. doi:10.1136/bjsm.2010.075085

Sahlén A, Winter R, Lind B, Jacobsen PH, Ståhlberg M, Marklund T, Fux T, Svensson J, Braunschweig F (2008) Magnitude, reproducibility, and association with baseline cardiac function of cardiac biomarker release in long-distance runners aged $>$ or $=55$ years. Am J Cardiol 102:218-222. doi:10.1016/j.amjcard. 2008.03.042

Scharhag J, Herrmann M, Urhausen A, Haschke M, Herrmann W, Kindermann W (2005) Independent elevations of N-terminal pro-brain natriuretic peptide and cardiac troponins in endurance athletes after prolonged strenuous exercise. Am Heart J 150:1128-1134. doi:10.1016/j.ahj.2005.01.051

Scharhag J, Urhausen A, Schneider G, Herrmann M, Schumacher K, Haschke M, Krieg A, Meyer T, Herrmann W, Kindermann W (2006) Reproducibility and clinical significance of exerciseinduced increases in cardiac troponins and $\mathrm{N}$-terminal pro brain natriuretic peptide in endurance athletes. Eur J Cardiovasc Prev Rehabil 13:388-397. doi:10.1097/00149831-200606000-00015

Scharhag J, George K, Shave R, Urhausen A, Kindermann W (2008) Exercise-associated increases in cardiac biomarkers. Med Sci Sports Exerc 40:1408-1415. doi:10.1249/MSS.0b013e318172 cf22

Serrano-Ostáriz E, Legaz-Arrese A, Terreros-Blanco JL, LópezRamón M, Cremades-Arroyos D, Carranza-García LE, Izquierdo-Alvarez S, Bocos-Terraz P (2009) Cardiac biomarkers and exercise duration and intensity during a cycle-touring event. Clin J Sport Med 19:293-299. doi:10.1097/JSM.0b013e3181 ab3c9d

Serrano-Ostáriz E, Terreros-Blanco JL, Legaz-Arrese A, George K, Shave R, Bocos-Terraz P, Izquierdo-Álvarez S, Bancalero JL, Echavarri JM, Quilez J, Aragonés MT, Carranza-García LE (2011) The impact of exercise duration and intensity on the release of cardiac biomarkers. Scand J Med Sci Sports 21:244-249. doi:10.1111/j.1600-0838.2009.01042.x

Shave R, George K, Gaze D (2007a) The influence of exercise upon cardiac biomarkers: a practical guide for clinicians and scientists. Curr Med Chem 14:1427-1436. doi:10.2174/0929867077 80831177

Shave R, George KP, Atkinson G, Hart E, Middleton N, Whyte G, Gaze D, Collinson PO (2007b) Exercise-induced cardiac 
troponin $\mathrm{T}$ release: a meta-analysis. Med Sci Sports Exerc 39:2099-2106. doi:10.1249/mss.0b013e318153ff78

Shave R, Ross P, Low D, George K, Gaze D (2010) Cardiac troponin $\mathrm{I}$ is released following high-intensity short-duration exercise in healthy humans. Int J Cardiol 145:337-339. doi:10.1016/j.ijcard. 2009.12.001

Stegmann H, Kindermann W, Schnabel A (1981) Lactate kinetics and individual anaerobic threshold. Int J Sports Med 2:160-165. doi: 10.1055/s-2008-1034604

Trivax JE, Franklin BA, Goldstein JA, Chinnaiyan KM, Gallagher MJ, deJong AT, Colar JM, Haines DE, McCullough PA (2010) Acute cardiac effects of marathon running. J Appl Physiol 108:1148-1153. doi:10.1152/japplphysiol.01151.2009

Wendel-Vos GC, Schuit AJ, Feskens EJ, Boshuizen HC, Verschuren WM, Saris WH, Kromhout D (2004) Physical activity and stroke. A meta-analysis of observational data. Int J Epidemiol 33:787-798. doi:10.1093/ije/dyh168

Whyte GP (2008) Clinical significance of cardiac damage and changes in function after exercise. Med Sci Sports Exerc 40:1416-1423. doi:10.1249/MSS.0b013e318172cefd

Wilson M, O'Hanlon R, Prasad S, Oxborough D, Godfrey R, Alpendurada F, Smith G, Wong J, Basavarajaiah S, Sharma S, Nevill A, Gaze D, George K, Whyte G (2010) Biological markers of cardiac damage are not related to measures of cardiac systolic and diastolic function using cardiovascular magnetic resonance and echocardiography after an acute bout of prolonged endurance exercise. Br J Sports Med Jun 11 [Epub ahead of print]. doi:10.1136/bjsm.2009.064089 